\title{
Influence of Probe Measuring Positions on Aerodynamics of a Transonic Axial flow Compressor
}

\author{
Hongwei MA \\ School of Energy and Power Engineering, \\ Beihang University \\ mahw@buaa.edu.cn \\ Beijing, 100191, China
}

\author{
Asad Islam \\ School of Energy and Power Engineering, \\ Beihang University \\ asadislam@buaa.edu.cn \\ Beijing, 100191, China
}

\begin{abstract}
The pneumatic probe technology obtains the flow field parameters such as total pressure, static pressure and air flow angle by measuring the pressure value of the probe head. Due to its low price, easy implementation, stable and reliable measurement, it is widely used in aerospace. However, as a contact test method, the blocking effect caused by the probe inevitably interferes with the flow field, causing the performance degradation or even early stall. The blockage caused by the probe struts is often overlooked by researchers. As the airflow bypasses; the unsteady wake formed due to probe struts, may enhance the complexity of local region flow. This complex flow can be sometimes beneficial to improve, while can also reduce the rotor performance. The current article aims to study the influence of probe-strut measuring positions on the aerodynamics of transonic speed axial flow compressor rotor. The probe strut is modelled at rotorupstream on different axial positions. The results are analysed with respect to the mass flow at which the rotor is near-stall without the probe-strut. However, this mass flow rate is the normal working condition for all the with probe-strut cases. Results show that total pressure ratio, choking mass flow and efficiency decrease as the probe-stem is inserted into the flowfield. The velocity along the stream also rises significantly at the near axial probe-position which contributes to the total pressure loss in the axial compressor rotor and also degradation of its performance.
\end{abstract}

\section{INTRODUCTION}

The flow distortion is more complex when the measurement probe is located upstream of the cascade which ultimately affect the engine performance and stability. Lepicovsky (Lepicovsky, 2008), investigated the flow field distortions due to the aerodynamic probe. His results predicted that the probes mounted within the rotor passage distort the flow-field causing the static pressure and axial velocity to decrease. Coldrick et al. (Coldrick, Ivey, \& Wells, 2004) presented the results of their measurement study using pneumatic pressure probes. The CFD simulations of the steady, uniform flow field around the probe in a low speed compressor were presented. They concluded that the major contribution to the measurement errors is the blockage effect. Furthermore, to measure the flow field in the simulations, a large scale probe was coupled with CFD probe calibration in a low speed compressor, which led to the conclusion that majority of probe measurement contaminations were due to steady state disturbance effects but the pressure gradient in stator passage did not impact any measurement error. Another research (He, Ma, Ren, \& Xiang, 2012) analysed the effects on the axial compressor aerodynamic performance using the aerofoil probes. The aerofoil probes were installed at the stator leading edge of a 1.5 stage axial flow compressor, the presence of these probes had a negative influence on all operating points of the compressor. These probes also induced the stream wise vortices along both sides of the blade. However, the intensity of these vortices gradually decreased along the stream. Moreover, the vortex formation was greater at the blade pressure side, while was small at the blade suction side; at the mid operating point. However, at the near to stall point, the vortex at the pressure side was suppressed, while was significant at the suction side of the blade. The vortex and endwall boundary layer interaction caused the flow to separate in the hub corner region. They thus concluded that the aerofoil probe located at $6.5 \%$ blade span having the positive pitch of 15 degrees had an important role in the vortex evolution near the hub region.

Our research group at Beihang University (BUAA) has been focusing on effects of probe on the stall characteristics on a large scale low-speed axial compressor in the recent years. Prof. Ma et. al (Ma, Li, \& Wei, 2016) conducted experimental research about stall inception by placing the cylindrical probe at 50 percent chord upstream of the rotor LE. Their results included the dynamic compressor behaviour from pre stall to full stall with probe and without probe 
conditions. The stability margin was also reported to be lower after installation of probe support.

An experimental research (Ma, Li, \& Wei, 2014) was carried out to study the effect of probe support on the flow field of a low speed axial flow compressor. The probe strut, intruding to 50 percent blade span and at 50 percent axial chord upstream, was tested in the regions from $5^{\circ}$ to $32^{\circ}$ off the probe support in the rotational direction at the rotor outlet and measurement were taken with a 5-hole and a high response total pressure probe. Their results indicated that blockage effects for low speed compressor varies at different span-wise heights of the probe strut and also leading to negative impact. The wakes caused by strut also weakens the intensity of tip vortex.

The effects of non-uniform tip clearances on transonic speed compressor Rotor 37 were numerically reported in terms of compressor performance (Ma \& $\mathrm{Li}, 2008)$. The efficiency and pressure ratio were of increasing nature due to non-uniform tip clearances compared to datum tip clearance.

The research relating to different measuring positions (Ma \& Zhang, 2010) in a centrifugal compressor reports arranging virtual probes at different radial and circumferential positions at both inlet and outlet. The effects of these measuring positions on aerodynamic performance depict notable measuring deviation of pressure ratio and efficiency at some orientations.

Another related research is about the transonic speed axial compressor (Kamel, 2010) which numerically reports that the different diameter-probe struts at rotor upstream and different inlet-angles can lead to the formation of distinct shock waves as a result of blockage caused and also lead to performance degradation. Furthermore another research (Islam \& Parvez,
2016), indicated the drop in pressure ratio and efficiency of Rotor37 as a result of distortions due to different inlet incidence angles.

\section{METHODOLOGY}

The current article presents an influence of the measurement probe positions on the aerodynamic characteristics of a transonic axial flow compressor rotor. The three dimensional Reynolds averaged Navier Strokes equation with $\mathrm{k}-\epsilon$ turbulence model are discretized using the finite volume approach and solved on the hexahedral grids for fluidflow analysis. The convergence criteria indicated the inception of stall point by the last converged point. The numerical results of pressure ratio and efficiency for without-probe strut shows good agreement with the experimental data, at the design rotational speed of $17188.7 \mathrm{rpm}$. The velocity, total pressure and chocking mass flow variations are discussed for the different axial positions. Bladegen, Turbo-Grid, CFX-Pre, CFX-Solver, and CFX-Post were utilized for Blade profile creation, high quality hexahedral grids generation, boundary condition definition, numerical analysis, and post-processing, respectively.

Fig. 1 shows the meridonal view of the rotor blade with probe strut axial positions marked upstream of the rotor. While, 50\% circumferential location for the probe-strut was chosen, because the flow field is not significantly influenced at this optimal position as reported (Xiang, Ge, Hou, Gao, \& Yang, 2017). The cases discussed in the present study are described in Table 1. A total pressure value of $101325 \mathrm{~Pa}$ was applied at the Inlet (Station 1), while the static pressure was applied at outlet (Station 2) to develop the characteristic maps for the rotor, with and without the probe-strut cases.

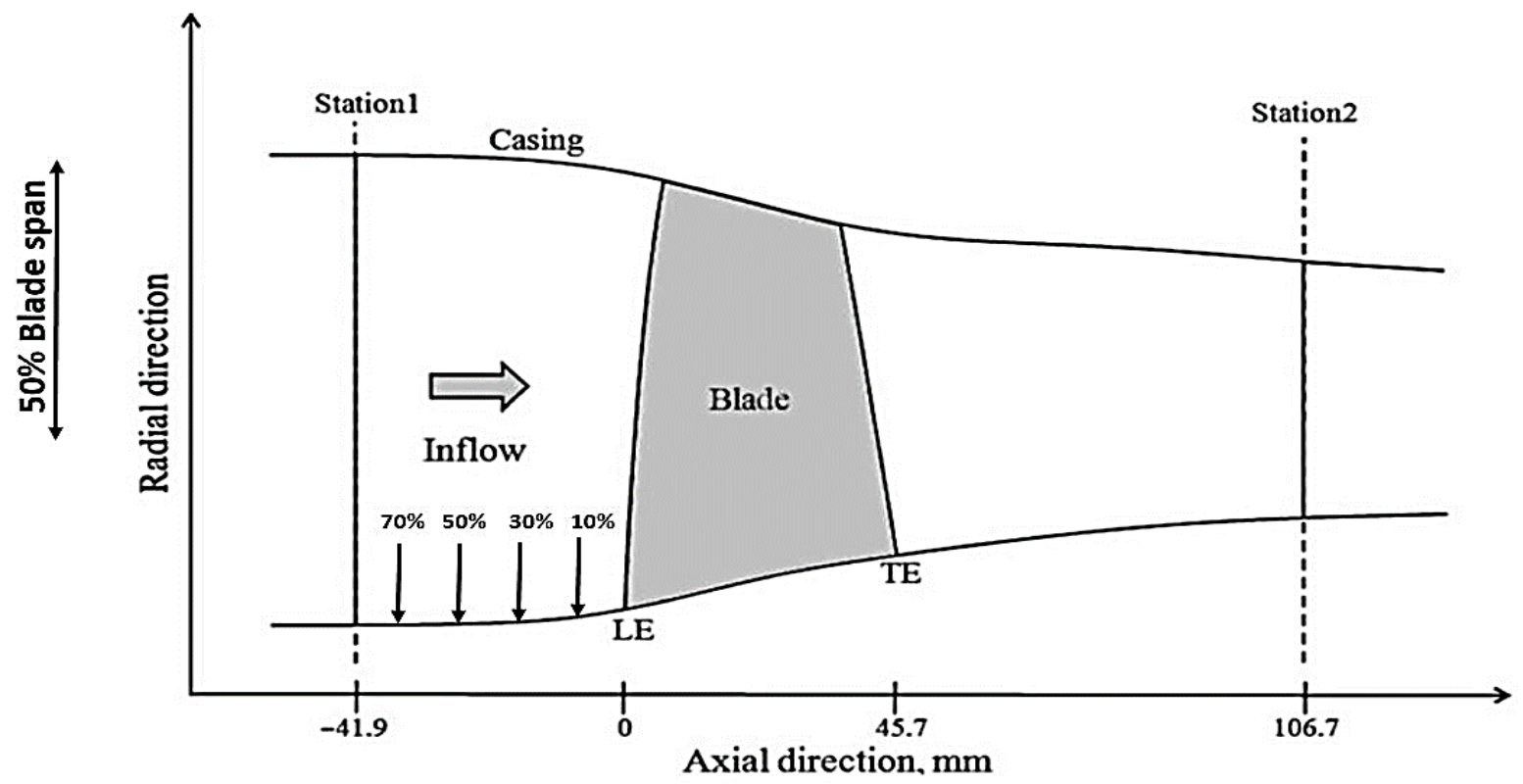

Figure 1: Probe axial positions and height 
Table 1: Description of cases

\begin{tabular}{|c|c|}
\hline Case (s) & Description \\
\hline Case A & $10 \%$ Axial Position, 50\% Probe height \\
\hline Case B & 30\% Axial Position, 50\% Probe height \\
\hline Case C & 50\% Axial Position, 50\% Probe height \\
\hline Case D & 70\% Axial Position, 50\% Probe height \\
\hline
\end{tabular}

\section{VALIDATION (NO PROBE SUPPORT)}

The transonic speed axial flow compressor with NASA Rotor 37 has an operational design speed of $17188.7 \mathrm{rpm}$ building a total pressure ratio of 2.056 at peak efficiency of 87.6 percent (Moore \& Reid, 1980). The blades are defined as the multiple circular arcs. The mesh consists of 1072236 nodes and 1001664 hexahedral elements. The characteristic map for the no-probe strut case were generated and validated with the experimental data (Moore \& Reid, 1980).

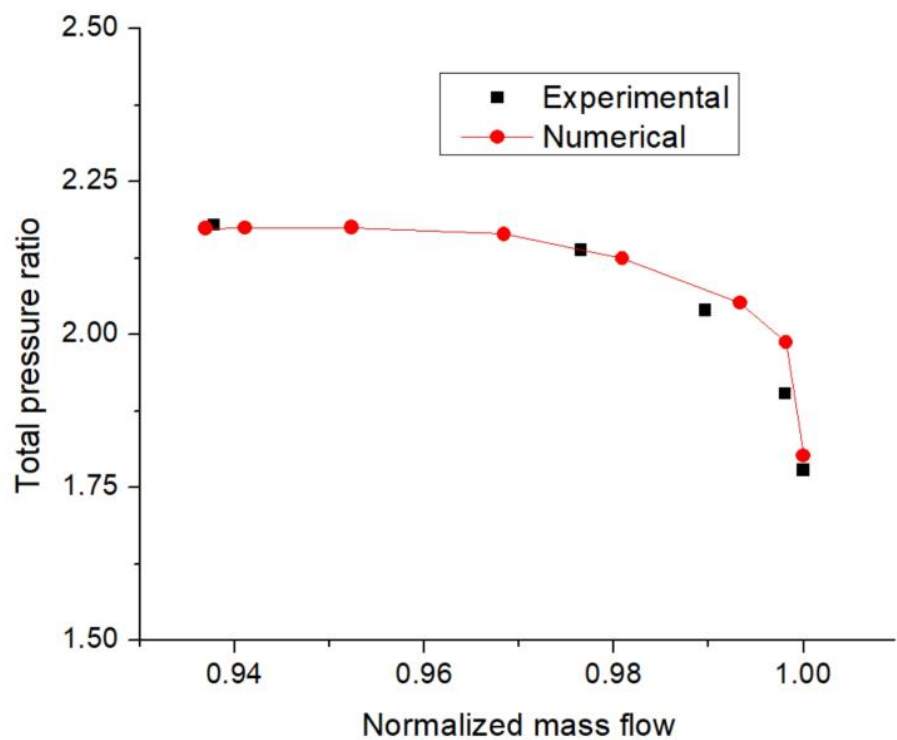

The results showed a good agreement between both the numerical and experiment data. Fig. 2 depicts the validation of numerical results at $100 \%$ rotational speed $(17188.7 \mathrm{rpm})$, and the mass flow rate is normalized by the choking mass flow rate. This validation thus forms a benchmark for further withprobe research.

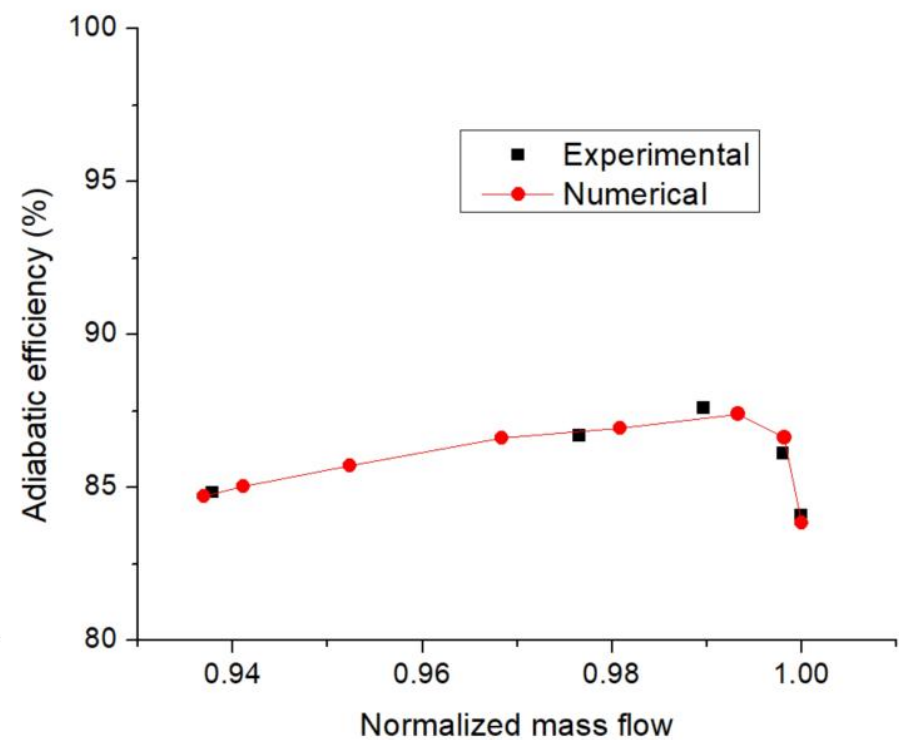

Figure 2: Characteristics map validation (a) Total Pressure ratio, (b) Adiabatic Efficiency

\section{WITH PROBE-STRUT CALCULATIONS}

The probe strut is modelled as a cylindrical rod of $8 \mathrm{~mm}$ in diameter. The full annulus consists of 36 rotor blades and 9 probe supports. Thus for computations, 40 degree section of the full wheel consisting of 4 rotor blades with 1 probe strut was simulated with periodic boundary conditions. The strut was placed at the rotor upstream on different axial locations and flow field of the rotor was analysed. The axial distances upstream of the rotor LE were considered as 10, 30, 50 and 70 percent of rotor blade chord length. While the probe height is 50 percent of blade span. (Fig.3). The support is modelled such that it intrudes from casing into the flow-field. The characteristic maps were generated and compared with the no probe case.

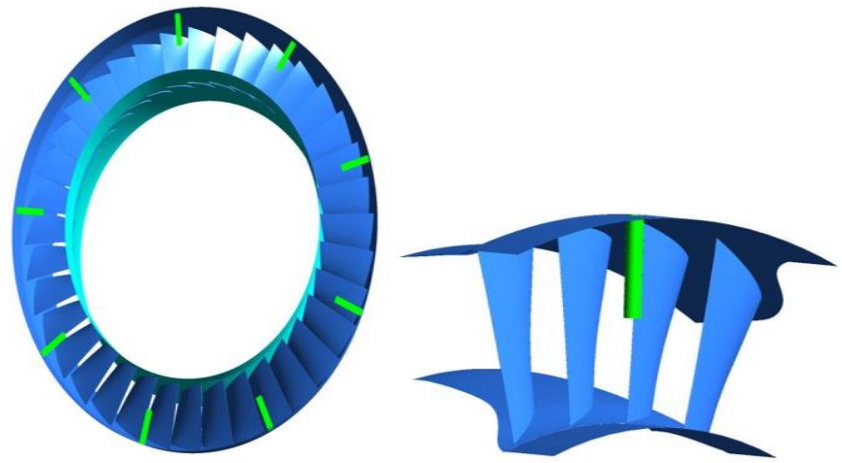

Figure 3: Probe-strut modelling at rotor upstream 


\section{RESULTS AND DISCUSSIONS}

\section{a) Total Pressure ratio variation}

When the probe-strut is intruded into the flow-field, it causes flow blockage and causes the total pressure ratio to decrease by 2.1 percent (Case D), as compared to no-probe case. The Fig.4 shows the total pressure ratio versus normalized mass flow rate (using choking mass flow of noprobe case). The pressure ratio significantly drops when the probe strut is located at 10 percent of axial-chord location.

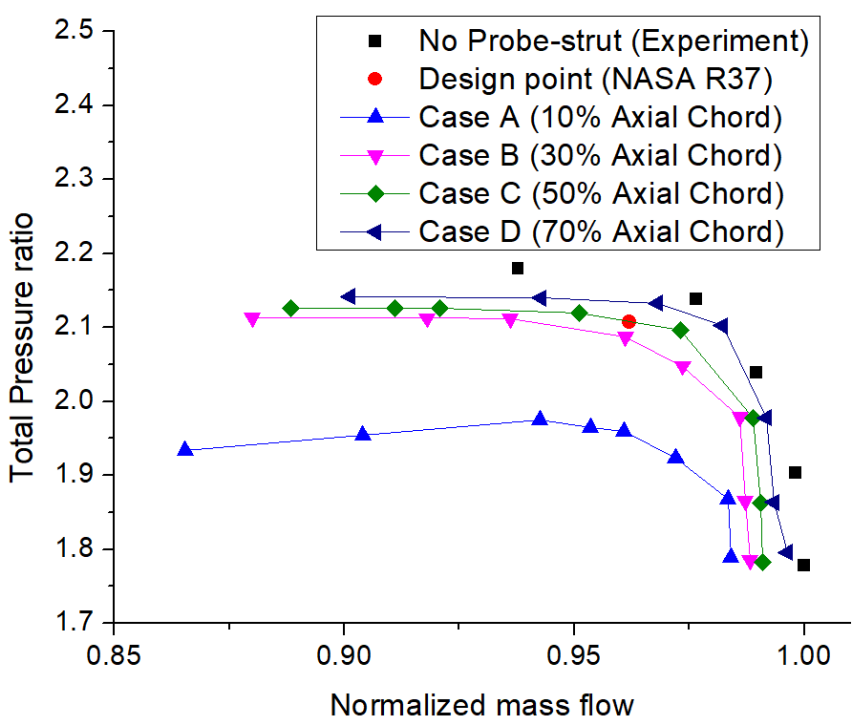

Figure 4: Total Pressure ratio (with probe-strut cases)
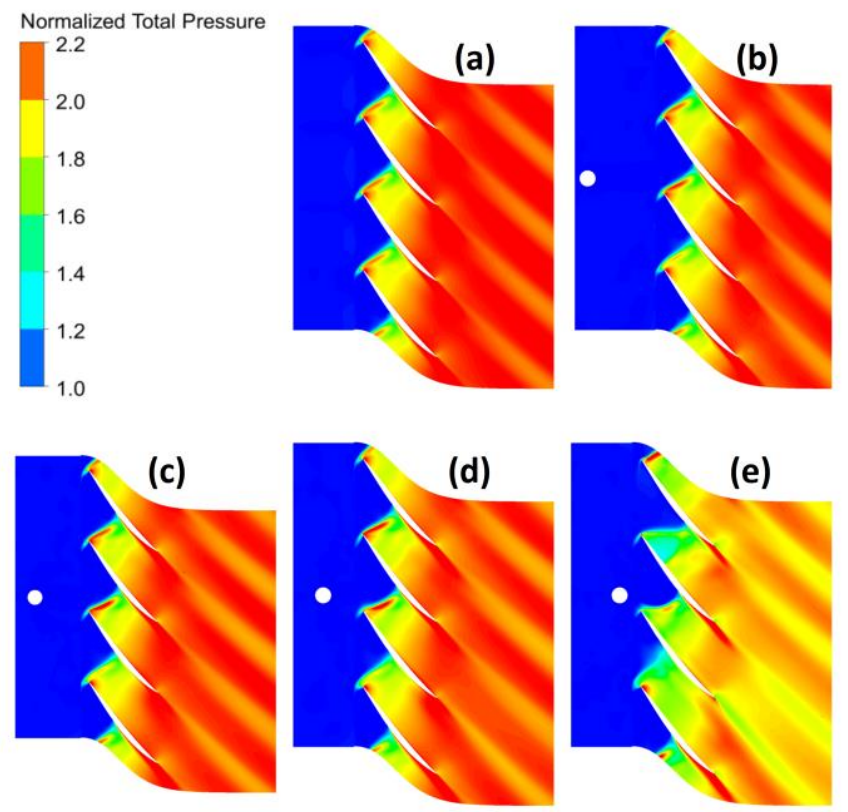

Figure 5:Total Pressure normalized contours (a) No probe, (b) Case D,(c) Case C,(d) Case B,(e) Case A
While in the other three cases, the drop is comparatively minimum.

Fig.5 depicts the normalized total pressure contours at half blade span. The total pressure is normalized by the inlet total pressure (Station 1, Fig.1). The contours show that the drop in total pressure for all the cases keeping the mass flow constant at which the without probe case is near to stall but all with-probe strut cases are normal working. The drop increases as the probe-strut is positioned closer and closer to the rotor leading edge. This drop in total pressure for all the cases keeping the no-probe support case as the reference is shown in Fig.6.

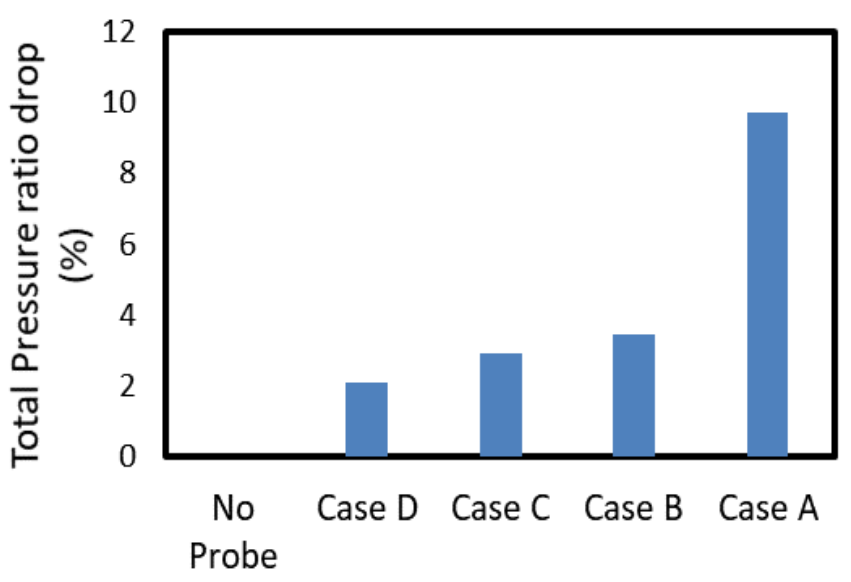

Figure 6: Total Pressure ratio loss at near-stall mass flow of clean flow case

\section{b) Adiabatic Efficiency}

Fig.7 shows that the adiabatic efficiency decrease after the probe insertion into the flow-field. The peak efficiency-

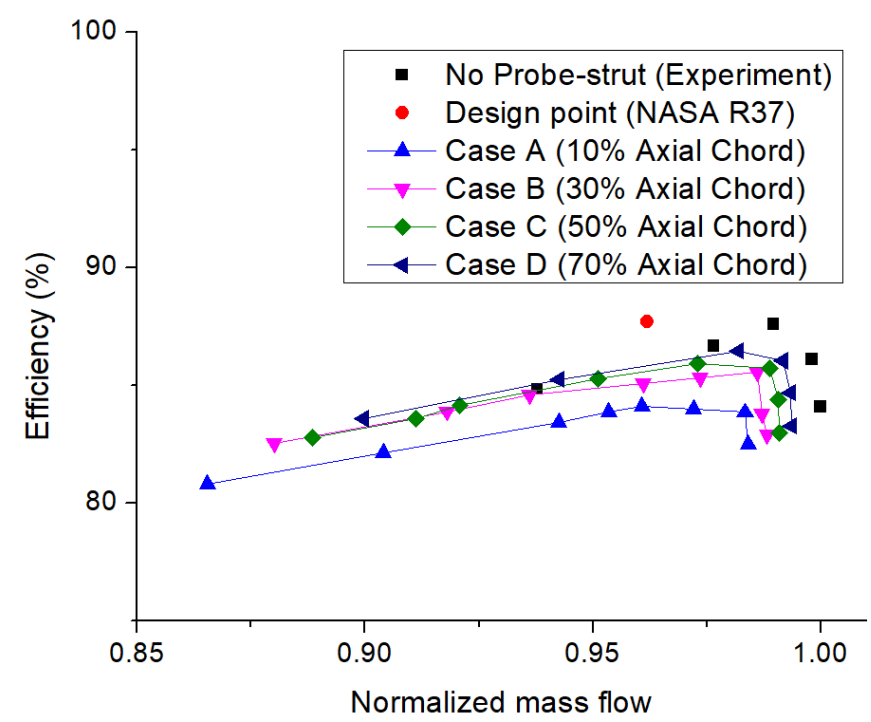

Figure 7: Efficiency for with-probe strut cases 
-point can be observed to be shifting to left. This shift is due to the loss in chocking mass flow (Fig.4,7\&11) with the change in axial position of the probe-strut. While for case A, which corresponds to the nearest axial position; the efficiency decreases by significant difference as compared to rest of the cases.

It also indicates the more-pressure build up on the rotor leading edge and highly disturbed situation in the case A.

Fig. 8 shows the increase and decrease of efficiency

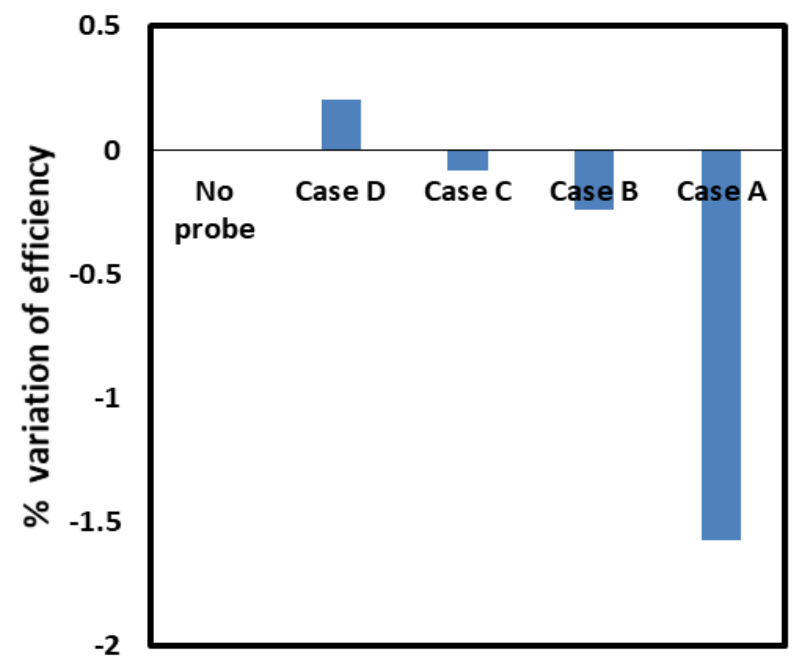

Figure 8: Adiabatic efficiency variation at nearstalling mass flow rate of without probe-strut case

-in percentages for all the cases keeping the no-probe case as the reference while also keeping the mass flow as constant. This mass flow is near-stall for no-probe case while is the normal working for all with-probe strut cases. At near position (Case A), the efficiency drop is $1.57 \%$ compared to no probe case along with a little drop in Case $\mathrm{C}$ of $0.08 \%$. Furthermore, a maximum increase of $0.2 \%$ was observed in Case $\mathrm{D}$ at this constant mass flow.

\section{c) Velocity}

The velocity variation for the different axial positions of the probe strut while also keeping constant mass flow. The velocity contours are normalized by the blade-tip velocity of $454 \mathrm{~m} / \mathrm{s}$ and the variation is shown on half of the blade span. This mass flow is the near stalling mass flow for the no-probe strut case while is the normal working mass flow for the rest of the cases, as shown in the characteristics maps Fig.4,7. The contours depict an increase of rotor-out flow velocity by relative positioning of strut closer to the rotor leading edge. However the presence of probe-strut also causes the relative decrease in intensity of maximum-velocity region at the blade LE in-front of it (Blade \#3 from top).

The significant increase in the rotor-out velocity at the near position of probe causes the total pressure to drop as depicted in the Fig.6.
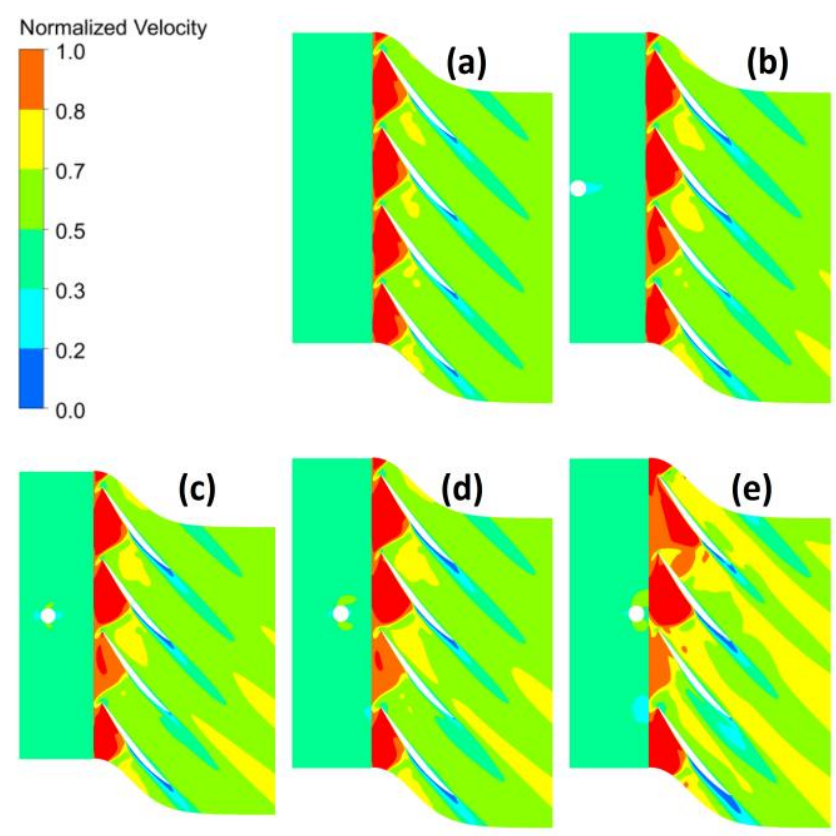

Figure 9: Normalized Velocity contours (a) No Probe-strut, (b) Case D, (c) Case C, (d) Case B, (e) Case A

The velocity streamlines showing the circulations occurring behind the probe-strut are depicted in fig.10. The circulating flow behind the cylindrical probe-strut indicates the very less or no vortical flow in case $\mathrm{A}$ and its immediate mixing with the high speed rotor flow.

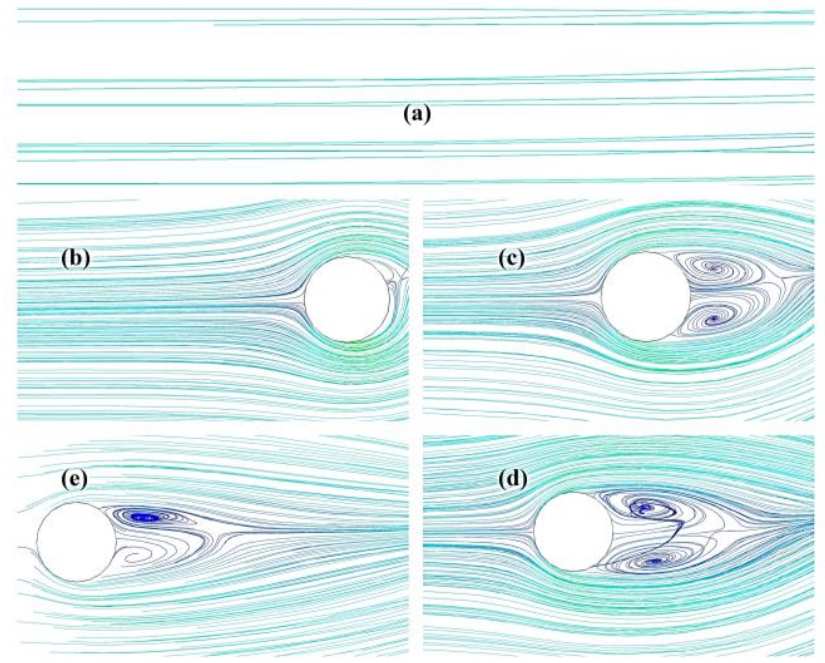

Figure 10: Velocity streamlines behind the probestrut (a) No Probe-strut, (b) Case A, (c) Case B, (d) Case C, and (e) Case D

\section{d) Choking mass flow}

Choking mass flow also drops as the probe strut is located near the rotor LE. Fig. 11 shows the drop in choked mass flow in percentage versus the cases described in Table 1. It indicates a drop of $0.36 \%$ at the far position (Case D) while 
$1.59 \%$ in near position (Case A). This decrease can be justified by the velocity patterns forming behind the probe that indicates the intensity of vortical flow. Case A shows no formation of vortices behind the strut due to very immediate mixing of flow behind the probe strut with the rotating rotor flow.

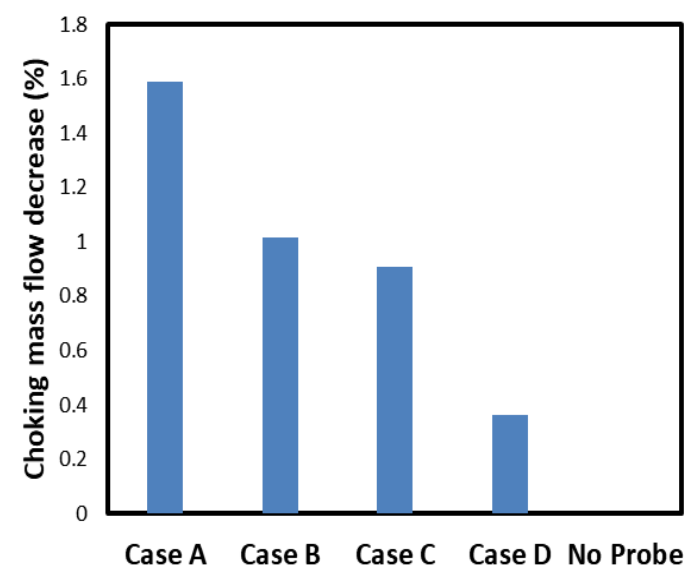

Figure 11: Choking mass flow decrease

\section{CONCLUSIONS}

This study focused on the influence of probe-strut axial positions on the axial compressor rotor performance. Thus concluding that the probe strut axial-positions has the influence on the aerodynamic characteristics of this high speed rotor aerodynamic performance. The following conclusions can be drawn from the study:

1. Case A, being very near position upstream of rotor leading edge, seems not-suitable for measurement because of poor rotor performance characteristics.

2. The stalling mass flow rate of clean-flow is the normal working mass flow for all the with probe-strut cases as depicted in the characteristics map.

3. The total pressure ratio decrease in all the cases with probe-strut insertion into the flow-field having a maximum loss of about $9.6 \%$ at the near position (Case A) and a minimum of $2 \%$ at far position (Case D), Fig.6.

4. At constant mass flow of no-probe strut case, the efficiency decrease by $1.57 \%$ at near position (Case A). A very little decrease of $0.08 \%$ in Case C; However, a maximum increase of $0.2 \%$ efficiency was observed in case D, Fig. 8 .

5. Drop in choking mass flow was also seen as the axial measurement location of the probe strut changes, with a maximum of $1.59 \%$ decrease in case A and a minimum of $0.36 \%$ in far position (case D).

6. The characteristics map (Fig 4,7) shows that the design point total pressure ratio of the Rotor 37 can be achieved by placing the probe at $50 \%$ axial-chord upstream of rotor LE.

7. The pressure increases on the rotor leading edge as the probe is positioned closer and closer to the rotor leading edge which causes the rotor-out velocity to increase resulting in the total pressure drop and also degraded rotor performance.

\section{REFERENCES}

Coldrick, S., Ivey, P. C., \& Wells, R. G. (2004). The Influence of Compressor Aerodynamics on Pressure Probes: Part 2 - Numerical Models. Volume 2: Turbo Expo 2004, 515-520. https://doi.org/10.1115/GT200453241

He, X., Ma, H., Ren, M., \& Xiang, H. (2012). Investigation of the Effects of Airfoil-probes on the Aerodynamic Performance of an Axial Compressor. Chinese Journal of Aeronautics, 25(4), 517-523. https://doi.org/10.1016/S1000-9361(11)60415-9

Islam, A., \& Parvez, K. (2016, March). Effects of Inlet Distorted Flows on the Performance of an Axial Compressor. Presented at the 18th International Conference on Mechanical, Industrial, and Manufacturing Engineering, Paris, France. https://doi.org/10.5281/zenodo.1111703

Kamel, M. K. (2010). Effects of Probes on Aerodynamic Performance of an Aero-Engine Compressor (Doctoral Thesis). Beihang University, Beijing, China.

Lepicovsky, J. (2008). Effects of a Rotating Aerodynamic Probe on the Flow Field of a Compressor Rotor (No. NASA/CR-2008-215215; pp. 1-27). Retrieved from ASRC Aerospace Corporation website: https://ntrs.nasa.gov/archive/nasa/casi.ntrs.nasa.gov/ 20080030792.pdf

Ma, H., \& Li, B. (2008). Effects of axial non-uniform tip clearances on aerodynamic performance of a transonic axial compressor. Journal of Thermal Science, 17(4), 331-336. https://doi.org/10.1007/s11630-008-0331-3

Ma, H., Li, S., \& Wei, W. (2014). Effects of probe support on the flow field of a low-speed axial compressor. Journal of Thermal Science, 23(2), 120-126. https://doi.org/10.1007/s11630-014-0685-7

Ma, H., Li, S., \& Wei, W. (2016). Effects of probe support on the stall characteristics of a low-speed axial compressor. Journal of Thermal Science, 25(1), 4349. https://doi.org/10.1007/s11630-016-0832-4

Ma, H., \& Zhang, J. (2010). Effects of measuring positions on the measured aerodynamic performance of a centrifugal compressor. Journal of Thermal Science, 19(2), 110-114. https://doi.org/10.1007/s11630-0100110-9

Moore, R. D., \& Reid, L. (1980). Performance of Single-Stage Axial-Flow Transonic Compressor with Rotor and Stator Aspect Ratios of 1.19 and 1.26 respectively, and with Design Pressure ratio of 2.05 (Technical Paper No. TP-1659). National Aeronautics and Space Administration Lewis Research Center.

Xiang, H., Ge, N., Hou, M., Gao, J., \& Yang, R. (2017). An experimental investigation of the effects of upstream probe disturbance on compressor cascade performance. Proceedings of the Institution of Mechanical Engineers, Part G: Journal of Aerospace Engineering, 231(7), 1248-1257. https://doi.org/10.1177/0954410016652435 